\title{
A Metric for Selection of the Most Promising Rules
}

\author{
Pedro Gago ${ }^{1,2}$ and Carlos Bento ${ }^{2}$ \\ 1 Escola Superior de Tecnologia e Gestão do Instituto Politécnico de Leiria \\ Morro do Lena, Alto Vieiro, 2400 Leiria \\ pgago@estg.iplei.pt \\ 2 CISUC - Centro de Informática e Sistemas da Universidade de Coimbra \\ Polo II, 3030 Coimbra \\ pgago, bento@eden.dei.uc.pt
}

\begin{abstract}
The process of Knowledge Discovery in Databases pursues the goal of extracting useful knowledge from large amounts of data. It comprises a pre-processing step, application of a data-mining algorithm and post-processing of results. When rule induction is applied for datamining one must be prepared to deal with the generation of a large number of rules. In these circumstances it is important to have a way of selecting the rules that have the highest predictive power. We propose a metric for selection of the $n$ rules with the highest average distance between them. We defend that applying our metric to select the rules that are more distant improves the system prediction capabilities against other criteria for rule selection. We present an application example and empirical results produced from a synthesized data set on a financial domain.
\end{abstract}

\section{Introduction}

The process of Knowledge Discovery in Databases pursues the goal of extracting useful knowledge from large amounts of data. It comprises a pre-processing step, application of a data-mining algorithm and post-processing of the results.

When rule induction is applied for data-mining one must be prepared to deal with the generation of a large number of rules. In these circumstances it is important to have a way of selecting the rules that have the highest predictive power.

The selection of interesting rules faces an essentially subjective problem. It is not likely that two different users will find the same rules to be interesting. Some authors support decisions on interestingness on subjective information provided by the user (Liu, Hsu and Chen [4], Klementinen, Mannila, Ronkainen, Toivonen and Verkamo [3]; Piatesky-Shapiro and Matheus [7]; Silberschatz and Tuzhilin [8]), other authors (Kamber and Shinghal [2]; Piatesky-Shapiro [6]; Major and Mangano [5]; Srikant and Agrawal [9]) choose to look for objective measures for-rule interestingness. Some of those measures are simplicity, statistical significance, coverage and confidence. 
Within our work the goal is not to decide on the individual interestingness of rules. Our aim is to build a set of rules that together gives a good coverage of the search space. We build on work on creativity (Gomes, Bento, Gago and Costa [1]) where a measure for the distance between cases is developed. We propose a metric for distance between two rules and use it to select the most heterogeneous set of rules that is possible in the assumption that this set has high predictive capabilities.

We present an example on a database of fictional data on bank clients. From the client database, rules are generated describing the 'good' and 'bad' clients. We use our metric on the rules to select $n$ heterogeneous rules supposed to be the $n$ rules that globally have the highest prediction power. Finally we present the results obtained using these rules and compare them with the use of the same number of rules selected randomly.

\section{A Metric for Distance Between Two Rules}

The rules are of the form 'IF conditions THEN conclusion'. Conditions is a conjunction of terms in the form $a_{1} s_{1} v_{1} \wedge \ldots \wedge a_{n} s_{n} v_{n}$ with $\mathrm{A}=\left\{\mathrm{A}_{1}, \ldots, A_{t}\right\}$ a set of attributes and $\mathrm{S}=\{<,<=,>\rangle=,,=\}$ a set of comparison operators, $a_{i} \in \mathrm{A}, s_{i} \in \mathrm{S}$ and $v_{i}$ being a numeric value. Conclusion is a class $c_{i} \in \mathrm{C}$ with $\mathrm{C}=\left\{c_{1}, \ldots, c_{m}\right\}$, a set of classes.

The measure we propose for distance between two rules with the same conclusion is based on three factors:

1. the number of attributes in one rule and absent in the other,

2. the number of attributes in both rules with overlapping values,

3. the number of attributes in both rules with values slight or null overlapping.

Based on these features we propose the following distance metric:

$$
\operatorname{dist}\left(r_{i}, r_{j}\right)= \begin{cases}\frac{\alpha \# D A_{i, j}+\beta \# D V_{i, j}-\omega \# E V_{i, j}}{\# F_{i}+\# F_{j}} & \text { if } \# N O_{i, j}=0 \\ 2 & \text { otherwise }\end{cases}
$$

In this metric we have:

\#DA $_{i, j} \quad$ Number of attributes in rule $i$ and not in rule $j$ plus the number of attributes in rule $j$ and not in rule $i$.

$\# \mathrm{NO}_{i, j} \quad$ Number of attributes both in rule $i$ and in rule $j$ but with non overlapping values.

\#DV $\mathrm{DV}_{i, j} \quad$ Number of attributes both in rule $i$ and in rule $j$, but with slightly overlapping values (we consider an overlapping bellow $66 \%$ ).

\#EV $\mathrm{EV}_{i, j} \quad$ Number of attributes in both rules, with overlapping values (we consider an overlapping above $66 \%$ ).

$\# \mathrm{~F}_{i}+\# F_{j} \quad$ Number of attributes in rule $i$ plus the number of attributes in rule $j$. 
For attributes appearing in both rules we check the intersection of their values. If there is no intersection we know the two rules cannot be applied to the same cases and thus we assign a value of two for the distance between the rules. We chose value two as it is a value higher than the one assigned in any other situation. In the case we have an intersection in less than $66 \%$ of the range of the rules values we consider the respective attributes to behave as if they were different attributes. We consider an attribute in two rules to be equal if the intersection of values in these rules is over $66 \%$ of the range of possible values.

To illustrate this concept of overlapping attributes consider that we have the attribute $A$ appearing in two rules (Rule \#1: $A>=20$ AND $A<=70$, Rule \#2: $A>=40$ ): We can apply rule 1 when the values for attribute $A$ are between 20 and 70 and can apply rule 2 if the values for attribute A are over 40 (see Fig. 1). Consider that the upper and lower limit for this attribute value is, respectively, 0 and 100 .

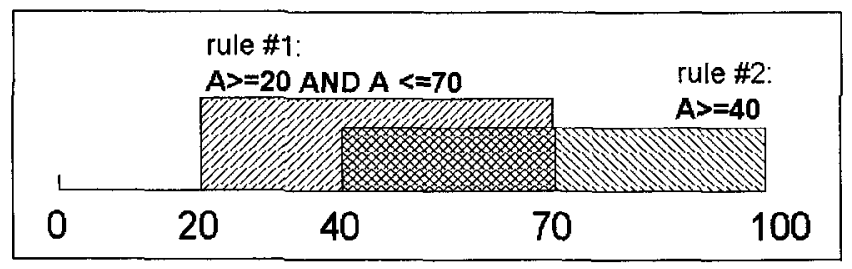

Fig. 1. Overlapping of an attribute in two rules.

We want to know if we can consider the conditions in both rules as equal. Suppose we have more rules with attribute $A$ and that the highest value attribute $A$ has in any of those rules is 100 . For attribute $A$ in rule 1 the range of possible values is $70-20=50$. In rule 2 the range is $100-40=60$ (100 is the maximum value for attribute A). The overlapping range for values in both rules is $70-40=30$. This overlapping happens in $30 / 50=60 \%$ of the range of possible values for attribute $A$ in rule 1 and in $30 / 60=50 \%$ of the range of possible values for attribute $\mathrm{A}$ in rule 2 . If we average the intervals of overlapping in the two rules we get $55 \%$. As this value is below $66 \%$ we consider attribute $\mathrm{A}$ in rule 1 and attribute $\mathrm{A}$ in rule 2 as if they were different attributes.

In the distance metric the characteristics which are strongly different between the two rules $\# \mathrm{DA}_{i, j}$ and $\# \mathrm{DV}_{i, j}$ ) increase the value returned by the function. The ones appearing in both rules $\left(\# \mathrm{EV}_{i, j}\right)$ decrease the function value. The terms $\# \mathrm{DA}_{i, j}, \# \mathrm{DV}_{i, j}$ and $\# \mathrm{EV}_{i, j}$ are weighed by constants $\alpha, \beta$ and $\omega$. We use $\alpha=1, \beta=2$ and $\omega=2$, making the metric take values between minus one and one. A value of one relates to two rules that have few things in common whereas a value of minus one indicates that the rules overlap strongly. When we are absolutely sure that the rules do not overlap the metric returns a value of two. 


\section{Rule Selection}

We use the metric described in the previous section for selection of the $n$ rules that provide the highest coverage for a data set. We pursue this goal by looking for the rules most different from the ones selected till now. We believe those rules to be the ones that provide, in general, the best coverage for the data set.

Considering the original set of classification rules to be $\mathrm{S}$ we must apply the following algorithm in order to build the set of $n$ rules which are more distant between each other. We use as distance criteria the one provided by the distance function described in the previous section. We name this set of rules $S_{R}$.

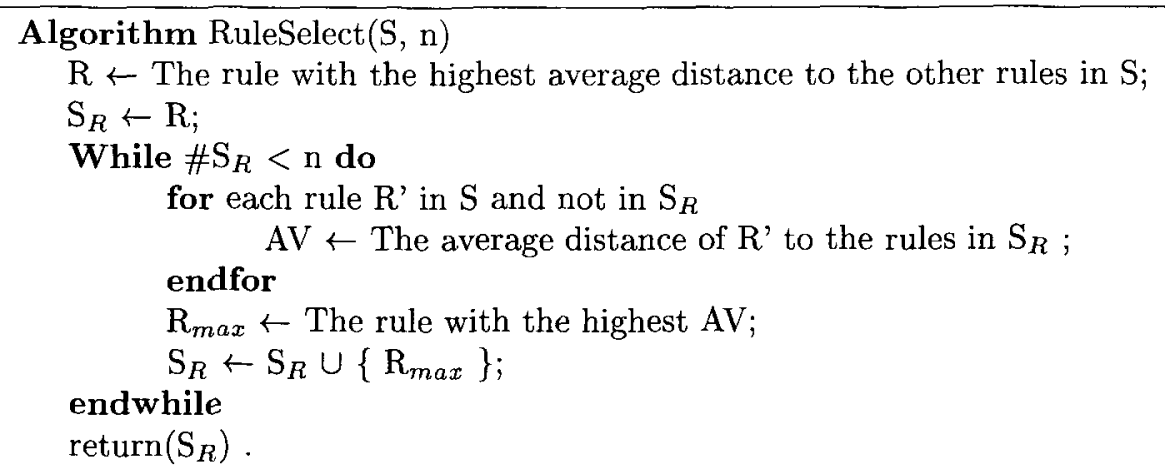

If we look for the rules with the lowest average distance we get the set of the rules closest to each other and we assume this set to be the least representative ruleset $\mathrm{S}_{L}$.

\section{Domain Description}

Our rule selection heuristic was tested in the domain of loan analysis. We synthesized a database with 3000 entries describing bank clients. The database was split in 2000 cases for training and 1000 for testing. For each client we know its yearly income, size of household, assets owned and amount to be paid per year. The database contains also information on whether the client presented a surety and on whether he is on a contract. The classification field in the database tells us if the client is a 'good' or 'bad' client. The following table shows the available information for three clients.

The independent attribute values were generated using the uniform distribution. The dependent attribute status is labeled 'Good' or 'Bad'. Status is assigned 'Good' or 'Bad' accordingly to two simple criteria: (1) if a client's available money (income minus loan) is over 500 units per household member then the client will honor his debts ((Income-Loan)/Household > 500); (2) 10\% of the clients on a contract are 'Bad' clients. We do not consider noisy data.

$\mathrm{C} 4.5$ is used on this database for rule generation. 
Table 1. Three cases

Income Household Assets Loan Surety Contract Status

\begin{tabular}{ccccccc}
\hline 7000 & 4 & 21000 & 3100 & No & No & Good \\
4100 & 1 & 3000 & 400 & No & No & Good \\
7500 & 4 & 8000 & 2300 & No & Yes & Bad \\
\hline
\end{tabular}

\section{An Example}

We considered the 14 rules generated by C4.5 (see appendix) for illustration of our approach. We describe how the distance between two rules is calculated on three rules in the set of 14 initial rules and show the results returned by the distance function (see Fig. 2).

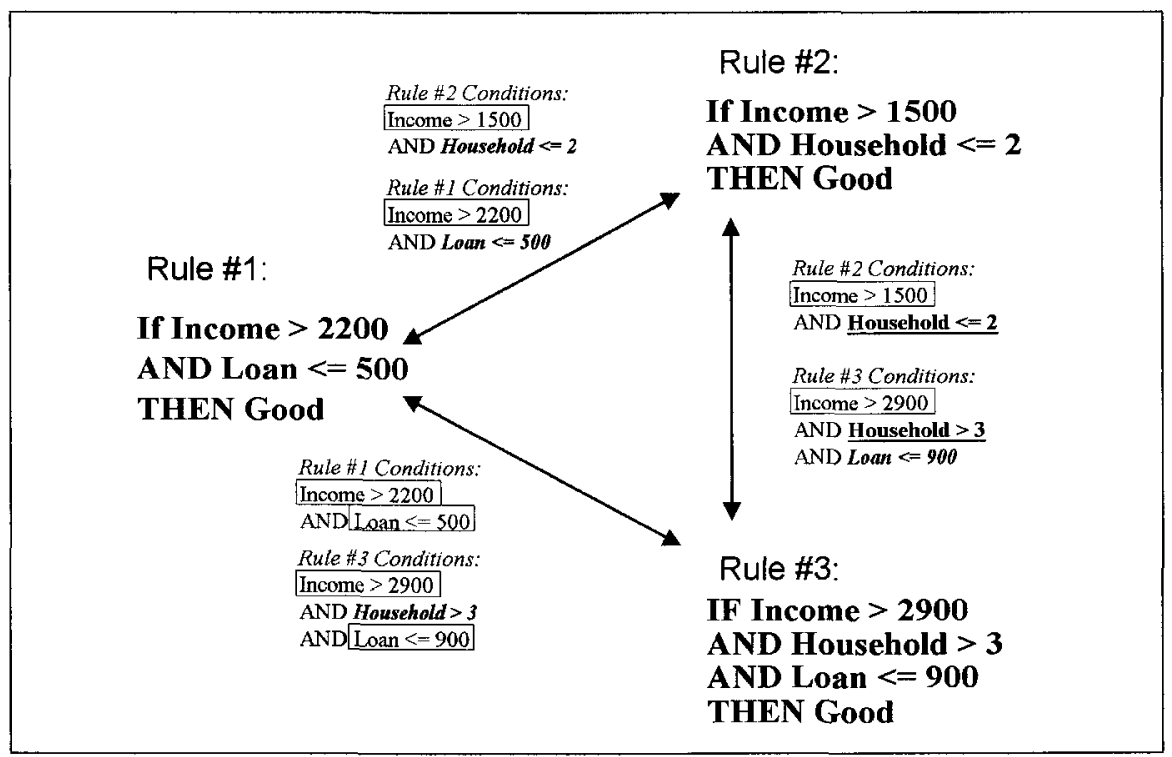

Fig. 2. Three rules generated by $\mathrm{C} 4.5$.

In order to determine the overlapping percentage for each attribute in the rules we need to know the range of values for this attribute. From the ruleset we have that the lowest value for Income is 1500 , the highest being 5800 . The Loan attribute ranges between 200 and 2900 .

Rules 1 and 2 have two distinct attributes - Loan and Household. The attribute Income appears in both rules. We have to determine the intersection for the values for this attribute.

Rule 1 may be used when Income is between 2200 and 5800 (the upper limit). For this rule the range is $5800-2200=3600$. For rule 2 the range is 
$5800-1500=4300$. Rules 1 and 2 overlap from 2200 to 5800 . The overlapping range of these rules is $5800-2200=3600$. The rules overlap in all the range of the first rule. The overlapping percentage for attribute Income in rule 1 is $3600 / 3600 * 100 \%=100 \%$. For rule 2 the overlapping takes place in $3600 / 4300 * 100 \%=83.7 \%$ of the range covered by the attribute Income. When we average these values we get $(100 \%+83.7 \%) / 2=91.85 \%$. As the value obtained is over $66 \%$ we consider the values in both rules to be equal.

We can now calculate the value returned by the distance function considering that $\# \mathrm{DA}_{1,2}=2$ (Household and Loan), $\# \mathrm{NO}_{1,2}=0, \# \mathrm{DV}_{1,2}=0, \# \mathrm{EV}_{1,2}=1$ and $\# \mathrm{~F}_{1}+\# \mathrm{~F}_{2}=4$.

The value returned for $\operatorname{dist}\left(\mathrm{r}_{1}, \mathrm{r}_{2}\right)$ is:

$$
\operatorname{dist}\left(r_{1}, r_{2}\right)=\frac{2+2 * 0-2 * 1}{4}=\frac{2-2}{4}=0
$$

For rules 1 and 3 we have $\# \mathrm{DA}_{1,3}=1$ (Household) and we must check to see if the values in the attributes Income and Loan should be considered equal or different. For Income in rule 1 the range is 3600 and in rule 3 the range is 2900 . The two rules overlap from 2900 to 5800 (the overlapping range is 2900). The overlapping percentage for rule 1 is $80.5 \%(2900 / 3600 * 100 \%)$ and for rule 3 is $100 \%(2900 / 2900 * 100 \%)$. Averaging these values we get $90.25 \%$. As the value is over $66 \%$ we consider the values to be equal.

For the other attribute shared by rules 1 and 3 (Loan) we have that the range for rule 1 is $500-200=300$ and for rule 2 is $900-200=700$ (200 is the lower limit for this attribute). The overlapping range is 300 . For rule 1 the overlapping percentage is $100 \%$ and for rule 3 it is $42.8 \%$. As the average for these values ( $71.4 \%)$ is once again over $66 \%$ we consider the values to be equal.

So, we have $\# \mathrm{DA}_{1,3}=1, \# \mathrm{DV}_{1,3}=0, \# \mathrm{EV}_{1,3}=2, \# \mathrm{OV}_{1,3}=0$ and $\# \mathrm{~F}_{1}+\# \mathrm{~F}_{3}=5$, and the value returned by the function is:

$$
\operatorname{dist}\left(r_{1}, r_{3}\right)=\frac{1+2 * 0-2 * 2}{5}=\frac{1-4}{5}=-\frac{3}{5}
$$

The attribute Household appears both in rule 2 and rule 3 and it is easy to see that these rules do not overlap. For these rules $\# \mathrm{NO}_{2,3} \neq 0$ and the metric returns a value of two.

$$
\operatorname{dist}\left(r_{2}, r_{3}\right)=2
$$

Rules 1 and 2 share one attribute with values very much alike. Rules 1 and 3 have two attributes with almost equal values and only one that is different. These results agree with our intuitive knowledge that rule 2 is "much farther" from rule 3 than from rule 1 and that rules 1 and 3 are very close.

In our example, the rule with the highest average distance to the others is rule 2 (the average distance is one) and it will be the first in set $S_{R}$. The second rule in $S_{R}$ will be rule 3 as it is the rule with the highest distance to the rule already in $\mathrm{S}_{R}$ (rule 2). 


\section{Empirical Results}

We applied our algorithm for rule selection to 14 rules (see appendix) to get the set $S_{R}$ of the most promising rules. We also considered a set with the least representative rules $\mathrm{S}_{L}$ and several sets of rules chosen at random $\mathrm{S}_{R A}$.

Using our program we built sets $S_{R}$ and $S_{L}$ with sizes from 1 to 8 . Using the 1000 cases for testing we measured the coverage for $S_{R}$ and $S_{L}$ function of the number $n$ of rules in these sets. We also determined the average coverage of randomly chosen groups of rules denominated $\mathrm{S}_{R A}$. The results are presented in the graph in Fig. 3.

When considering the sets $\mathrm{S}_{R}, \mathrm{~S}_{R A}$ and $\mathrm{S}_{L}$ with a number $n$ of rules near half the number in the original ruleset we see that the rules in $S_{R}$ have a higher prediction power than those in $\mathrm{S}_{R A}$ and in $\mathrm{S}_{L}$. We also note that the rules in $\mathrm{S}_{L}$ are consistently the ones with the lowest predictive power.

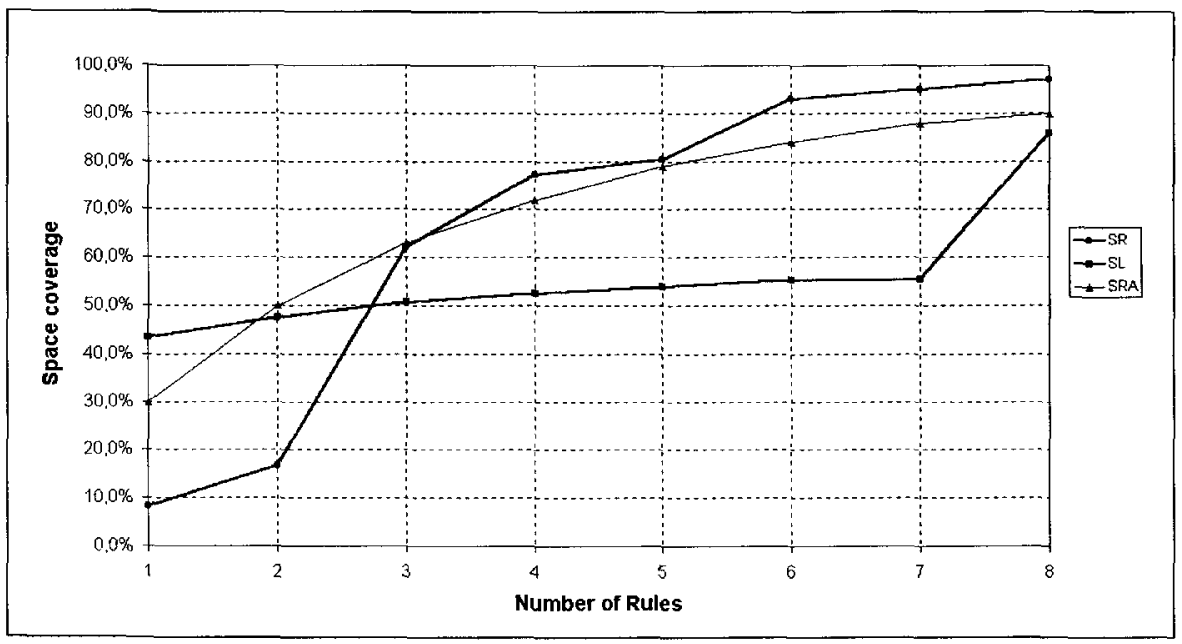

Fig. 3. Comparison of the sets coverage.

\section{Conclusions and Future Work}

We developed a method for rule selection that consistently outperforms random choice. Using this method one can reduce the time spent analyzing uninteresting rules and concentrate on the best ones. Unlike previous work (Kamber and Shinghal [2]; Piatesky-Shapiro [6]; Major and Mangano [5]; Srikant and Agrawal [9]) we do not try to measure the interestingness of a rule. We use our metric to measure the distance between any two rules in order to build a set with the rules with the highest predictive power. 
One of the problems found within our framework is the difficulty in the assignment of weights for our metric. For now this selection is guided by the fact that these values make the metric take values between minus one and one. We believe our approach is domain independent as long as the underlying data are uniformly distributed.

This method for rule selection works well if the underlying data follow a uniform distribution. If the data follow other distributions one must adjust the algorithm that allows us to calculate the range of the interception between two rules. One of the next steps is to adapt the metric to make it work with normally distributed data. It would also be interesting to explore the behavior of the metric when it has available domain knowledge concerning the upper and lower limits for the values of each attribute without having to guess them from the induced rules. For now our program looks at the rules in order to find values it may use as limits. We will probably get better results if we ask the user to provide us with an upper and lower limits for the attributes values.

\section{References}

1. Gomes, P., Bento, C., Gago, P., Costa, E.: Towards a Case-Based Model for Creative Processes. Proceedings of the $12^{\text {th }}$ European Conference on Artificial Intelligence (1996) 122-126.

2. Kamber, M., Shingal, R.: Evaluating the Interestingness of Characteristic Rules. Proceedings of the Second International Conference on Knowledge Discovery $\&$ Data Mining (1995) 263-266.

3. Klementinen, M., Mannila, H., Ronkainen, P., Toivonen, H., Verkamo, A.: Finding Interesting Rules from Large Datasets of Discovered Association Rules. Proceedings of the Third International Conference on Information and Knowledge Management (1994) 401-407.

4. Liu, B., Hsu, W., Chen, S.: Using General Impressions to Analyse Discovered Classification Rules. Proceedings of the Third International Conference on Knowledge Discovery \& Data Mining (1997) 31-36.

5. Major, J.A., Mangano, J.: Selecting Among Rules Induced from a Hurricane Database. Proceedings of the AAAI-93 Workshop on Knowledge Discovery in Databases (1993) 28-44.

6. Piatesky-Shapiro, G.: Discovery, Analysis and Presentation of Strong Rules. $G$. Piatesky-Shapiro \& W.J. Frawley, eds., Knowledge Discovery in Databases. Menlo Park, CA: AAAI/MIT Press. (1991) 229-248.

7. Piatesky-Shapiro, G., Matheus, C.J.: The Interestingness of Deviations. Proceedings of the AAAI-94 Workshop on Knowledge Discovery in Databases (1994) 25-36.

8. Silberschatz, A., Tuzhilin, A.: What Makes Paterns Interesting in Knowledge Discovery Systems. IEEE Trans. On Know. and Data Eng. 8(6) (1996) 970-974.

9. Srikant, R., Agrawal, R.: Mining Generalized Association Rules. Proceedings of the $21^{s t}$ VLDB conference (1995) 407-419 
Appendix - The rules generated by C4.5.

\begin{tabular}{|c|c|}
\hline $\begin{array}{l}\text { Rule \#1: } \\
\text { if Income }>5600 \\
\text { and Contract = } 0 \\
\text { then Good }\end{array}$ & $\begin{array}{l}\text { Rule \#9: } \\
\text { if Income }>1500 \\
\text { and Household }<=3 \\
\text { and Loan }<=200 \\
\text { then Good }\end{array}$ \\
\hline $\begin{array}{l}\text { Rule } \# 2 \text { : } \\
\text { if Income }>4100 \\
\text { and Assets }<=28000 \\
\text { and Surety }=0 \\
\text { and Contract }=0 \\
\text { then Good }\end{array}$ & $\begin{array}{l}\text { Rule \#10: } \\
\text { if Income }>1500 \\
\text { and Household }<=2 \\
\text { then Good }\end{array}$ \\
\hline $\begin{array}{l}\text { Rule \#3: } \\
\text { if Income > } 5800 \\
\text { then Good }\end{array}$ & $\begin{array}{l}\text { Rule \#11: } \\
\text { if Income }>2300 \\
\text { and Household }<=3 \\
\text { and Loan }<=1100 \\
\text { then Good }\end{array}$ \\
\hline $\begin{array}{l}\text { Rule \#4: } \\
\text { if Income }>3600 \\
\text { and Loan }<=1800 \\
\text { then Good }\end{array}$ & $\begin{array}{l}\text { Rule \#12: } \\
\text { if Income }>1900 \\
\text { and Household }<=3 \\
\text { and Loan }<=700\end{array}$ \\
\hline $\begin{array}{l}\text { Rule \#5: } \\
\text { if Income }>4500 \\
\text { and Loan }<=2900 \\
\text { then Good }\end{array}$ & $\begin{array}{l}\text { then Good } \\
\text { Rule \#13: } \\
\text { if Income }>2200 \\
\text { and Loan }<=500\end{array}$ \\
\hline $\begin{array}{l}\text { Rule \#6: } \\
\text { if Income }>3100 \\
\text { and Loan }<=1300 \\
\text { then Good }\end{array}$ & $\begin{array}{l}\text { then Good } \\
\text { Rule \#14: } \\
\text { if Household }<=1 \\
\text { then Good }\end{array}$ \\
\hline $\begin{array}{l}\text { Rule \#7: } \\
\quad \text { if Income }>2900 \\
\text { and Household }<=3 \\
\text { then Good }\end{array}$ & \\
\hline $\begin{array}{l}\text { Rule \#8: } \\
\text { if Income }>2900 \\
\text { and Household }>3 \\
\text { and Loan }<=900 \\
\text { then Good }\end{array}$ & \\
\hline
\end{tabular}

